\title{
Anesthesia Management of Electroconvulsive Therapy at the Late of Pregnancy: A Case Report
}

\author{
Heshmatolah Moosavi Rineh ${ }^{1}$, Hossein Khoshrang ${ }^{(\mathbb{}}{ }^{(}$, Cyrus Emir Alavi $^{{ }^{\circledR}}$, Siamak Rimaz $^{2^{\circledR}}$, Gelareh \\ Biazar $^{2 *}$, Reyhaneh Shahrokhi Rad ${ }^{2}$, Mahsa Khosousi Sani ${ }^{4}$
}

\begin{abstract}
Objectives: Major maternal depression is well known as a morbid and common disorder with a prevalence of $12 \%$ in the third trimester of pregnancy. Psychotropic medications might not be quite safe in these cases. In addition, pharmacological resistance and any life-threatening conditions can appear with the acute need for an instant therapeutic response. In these cases, electroconvulsive therapy (ECT) might be the only effective option. However, both clinicians and patients often feel hesitant to utilize ECT throughout pregnancy due to the lack of reliable literature.

Case Presentation: The challenging case of a 26-year-old pregnant woman at the 37 weeks of gestation was presented with major depression, who achieved full symptomatic remission after ECT. The critical status of this case was well managed by ECT treatment. Through the follow-ups, it was found that she is under medical therapy and has a normal life now. She is taking care of her baby and her symptoms are controlled as well.

Conclusions: Overall, ECT could be a safe and effective treatment at late pregnancy. Obviously, a multidisciplinary team approach is crucial for achieving the desired goals.

Keywords: Anesthesia, ECT, Pregnancy, Third trimester
\end{abstract}

\section{Introduction}

Many physiological and psychological changes occur during pregnancy which affect women's life and obviously require a proper adaptation (1). Prenatal depression with a prevalence of $12 \%$ to $36 \%$ as an independent risk factor for low birth weight and preterm labor could be a big challenge for psychiatrists $(2,3)$. In addition, treating maternal depression as an independent risk factor for low birth weight and preterm labor is considered a critical challenge for psychiatrics. If untreated, it might simultaneously result in several behavioral complications such as malnutrition, poor prenatal care, substance abuse, and even suicide and fetal harm. Pharmacological treatments induce severe adverse effects (4). Further, unresponsive pharmacological treatment, catatonia, extreme agitation, high suicide risk, or any other life-threatening conditions can emerge with the urgent need for a quick resolution. To manage these cases, electroconvulsive therapy (ECT) is regarded as the only valid treatment strategy $(5,6)$. According to scientific literature, maternal major depression responds well to ECT with a success rate of $84 \%$. However, it is often neglected due to the fear of harm for both the mother and fetus. Thus, the majority of studies have focused on medication versus ECT $(5,7)$. Contrary to previous studies, which indicated that ECT is a quite safe treatment throughout pregnancy, recent studies declare that ECT administration during pregnancy should be based on the point of whether potential risks outweigh the benefits $(5,8,9)$. There are some case reports regarding the use of ECT during pregnancy although it is rare in the last weeks and data on anesthesia management are limited as well. In early studies from 1942 to 1970 , ECT was even performed without anesthesia. Furthermore, most of the reports have failed to evaluate the newborn Apgar and no follow-up data are available on the children (8). Obviously, the ECT pattern may not put a difference between pregnant and non-pregnant women. However, managing anesthesia and providing facilities make differences in this regard (6). The present study focused on the anesthesia management of a challenging pregnant case with major depression who successfully underwent an ECT protocol.

\section{Case Presentation}

Her mood changes started during pregnancy, almost from the middle of the second trimester, thus she received psychotropic medication. Nearly at the late of the third trimester, her symptoms got worse and her body was resistant to pharmacologic treatments. In addition, the patient's status was deteriorated and she was hospitalized since she refused to continue medication. Then, she 
was consulted for ECT administration and visited for anesthesia preoperative evaluation. First, the informed consent was taken from her family and all potential risks and benefits for both mother and fetus were described as well. The obstetrician team was consulted with, and they recommended that ECT could be administrated only in an urgent situation if no other effective treatment option was accessible. Then, the anesthesiologist asked the responsible psychiatrics to document this urgency. Furthermore, the necessity of the attendance of the obstetric team was noted while performing the procedure and throughout the recovery time. She fasted for eight hours before the procedure. An hour before ECT administration, she received ranitidine $150 \mathrm{mg}$, metoclopramide $10 \mathrm{mg}$, and crystalloid $500 \mathrm{~mL}$ through an angiocath (18 gauge). As soon as she lied on the bed, a nurse pushed the pregnant uterus to the left to avoid supine hypotension. Further, standard monitoring was performed, including electrocardiogram, percent oxygen saturation, and noninvasive blood pressure before, during, and after the procedure. Fetal movement, reactivity, and heart rate were also monitored by an obstetrician. Pre-oxygenation with $\mathrm{O}_{2} 100 \%$ was performed for three minutes and all the equipment for emergency airway management was available. Given the patient weight of $80 \mathrm{Kg}$, anesthesia was induced by intravenous propofol $120 \mathrm{mg}$ immediately, followed by intravenous succinylcholine $60 \mathrm{mg}$ for muscle relaxation. Moreover, an alternative current was applied on both sides of the head when obtaining an appropriate depth of anesthesia. To suppress propofol injection pain, the venous drainage of the upper arm was occluded by an air-filled tourniquet. Then, a low dose of propofol as 15 mg was injected, followed by properly ventilating the lungs with a face mask and bag using 100\% oxygen. During the procedure, the hemodynamic status remained stable and she was transferred back to the psychiatric inpatient ward after confirming full recovery. After each session, she was advised to call her obstetrician if feeling any contractions, vaginal bleeding, or any changes in fetus movements. A significant fetal heart rate reduction was observed at the second session and both the anesthesiologist and obstetrician were well-prepared for an urgent caesarian section, but it reached a normal base before the need for any intervention. She also experienced intermittent contraction. However, the obstetrician was not sure that it was due to ECT and preferred a close observation and fetal monitoring while refusing to terminate the pregnancy because she believed that there was no exact and legal indication for an elective delivery. The conditions got more and more difficult session by session. For the next ECT sessions, she received a prophylactic dosage of magnesium sulfate as the tocolytic agent in order to prevent uterine contractions. Fortunately, her poor conditions improved after three sessions and she reported a significant decrease in her depression feeling. She underwent 6 ECT sessions, which were conducted over a two-week period (3 sessions per week). Finally, she successfully finished her treatment course and symptomatic recovery was obtained completely. Within this period, no abnormality was detected in fetal monitoring except for a transient episode of Fetal heart reduction (FHR). After the termination of the ECT course and when she was 39 weeks and 4 days of gestation, she was admitted because of contractions and a healthy male newborn with an Apgar score of 9 was born via a spontaneous vaginal delivery. Medical therapy was started and she was recommended to be closely followed up because her symptoms might be refractory and she would experience the same conditions again.

\section{Discussion}

The anesthesia management of pregnant women undergoing ECT is challenging for both anesthesiologists and psychiatrists since short and safe anesthesia is required for providing a high-quality grand mal seizure. In addition, the anesthesiologist should be well-prepared for resuscitation and emergency cesarean section if necessary $(6,10)$. Indeed, updated studies do not strongly support the definite statement of previous studies, which indicated that ECT was "low risk and high efficacy in the management of specific disorders in all three trimesters of pregnancy" (11). In other words, the voices of concern are heard in recent review studies due to procedure-related complications including fetal hip dysplasia, premature birth and meconium peritonitis, fetal hypoxia, fetal cardiac arrhythmias, slight vaginal bleeding, abdominal pain, and uterine contractions and ECT during pregnancy is only recommended when other strategies are impossible or insufficient $(8,12)$. Anderson and Reti reviewed 57 studies, in which 339 pregnant women received ECT, and reported a low number of 11 neonatal abnormalities (13). Later, in a review article including 67 case reports from 1970 to 2013, in which 169 pregnant women received ECT, Leiknes et al found that most cases were in the second trimester with the main diagnosis of depression/bipolar disorder. It was noticeable that one-third of these cases were affected by ECT-related adverse events including premature births, uterine contractions, and fetal heart rate reduction. A baby mortality rate of $7.1 \%$ was reported as well. They concluded that ECT during pregnancy must be only selected as the last solution $(4,8)$. Although it was unusual to administer ECT at this late pregnancy and inadequate literature with conflicting reports was available, it was a proper decision making because no other option was left. In this case, the emergency should be questioned and the urgent necessity of ECT must be documented by the responsible psychiatrics due to the high risk of the last days of pregnancy. Furthermore, consultation with the obstetrician should be done because they might plan an elective delivery in order to continue the treatment process in safer conditions. CT anesthesia management is extremely vital during pregnancy because some threatening conditions could be just prevented or 
alleviated by anesthesia considerations in addition to the common adverse effects like headache and myalgia (14). Studies have shown that ECT might induce early or delayed contractions. According to some reports, despite the urgent need for ECT, it was discontinued due to uterine contractions (4). When performing ECT, an increase occurs in uterine contractions due to oxytocin release (15) and warns the risk of premature birth (1). Transient hypertension and insufficient ability to metabolize succinylcholine are the other plausible explanations for increasing the uterine tone following ECT. In the management of this case, several attempts were made to minimize the mentioned contributing factors. FHR, as the most common adverse effect in ECT, is not easy to distinguish that is due to anesthetic agents or ECT intervention or both (16). Prolonged seizure duration results in a severe FHR reduction which leads to the need for an emergency caesarian section (17) thus anesthetic agents with the mentioned property should be avoided. Moreover, both left lateral tilt and adequate hydration could prevent placental perfusion diminution. Propofol was used as the induction drug because of the rapid onset and short recovery time and rapid redistribution before it passes to the placenta and receives the fetus (18). The other reason for choosing propofol was that severe fetal bradycardia was not reported following anesthesia induction. Another research also is in favor of propofol (19). In general, ECT could be a valuable and effective treatment modality in resistance and emergency cases at the late pregnancy and attempts should be made not to evacuate these cases from this valuable treatment option. This report also highlights the importance of a multidisciplinary approach for managing these cases, for instance, a highly-skilled team consisting of an anesthesiologist, psychiatrist, obstetrician, and trained ECT staff. Therefore, future studies are welcomed considering the importance of the issue and the significant progression in medical sciences.

\section{Conflict of Interests}

None of the authors had any conflict of interests regarding this paper.

\section{Ethical Issues}

Informed consent was obtained from the patient.

\section{Financial Support}

This study was not sponsored by any organization.

\section{Acknowledgments}

We would like to appreciate the efforts of Ms. Jalileh Massomi for editing the present paper.

\section{References}

1. Bulbul F, Copoglu US, Alpak G, et al. Electroconvulsive therapy in pregnant patients. Gen Hosp
Psychiatry. 2013;35(6):636-639. genhosppsych.2013.06.008

2. Coshal S, Jones K, Coverdale J, Livingston R. An overview of reviews on the safety of electroconvulsive therapy administered during pregnancy. J Psychiatr Pract. 2019;25(1):2-6. doi:10.1097/pra.0000000000000359

3. Brown K, Kathol D. Mood Disorders in Pregnancy: A Guide to Gender-based Issues in Evaluation, Diagnosis, and Treatment. Neurology and Psychiatry of Women; 2019:157-172. doi:10.1007/978-3-030-04245-5_18

4. Pesiridou A, Baquero G, Cristancho P, et al. A case of delayed onset of threatened premature labor in association with electroconvulsive therapy in the third trimester of pregnancy. J ECT. 2010;26(3):228-230. doi:10.1097/ YCT.0b013e3181c3aef3

5. Ray-Griffith SL, Coker JL, Rabie N, Eads LA, Golden KJ, Stowe ZN. Pregnancy and electroconvulsive therapy: a multidisciplinary approach. J ECT. 2016;32(2):104-112. doi:10.1097/yct.0000000000000297

6. Ward HB, Fromson JA, Cooper JJ, De Oliveira G, Almeida M. Recommendations for the use of ECT in pregnancy: literature review and proposed clinical protocol. Arch Womens Ment Health. 2018;21(6):715-722. doi:10.1007/ s00737-018-0851-0

7. Sinha P, Goyal P, Andrade C. A meta-review of the safety of electroconvulsive therapy in pregnancy. J ECT. 2017;33(2):81-88. doi:10.1097/yct.0000000000000362

8. Leiknes KA, Cooke MJ, Jarosch-von Schweder L, Harboe I, Høie B. Electroconvulsive therapy during pregnancy: a systematic review of case studies. Arch Womens Ment Health. 2015;18(1):1-39. doi:10.1007/s00737-013-0389-0

9. Karahan MA, Büyükfırat E1, Binici O, et al. The Effects of Rocuronium-sugammadex on Fetomaternal Outcomes in Pregnancy Undergoing Electroconvulsive Therapy: A Retrospective Case Series and Literature Review. Cureus. 2019;11(6):e4820. doi:10.7759/cureus.4820

10. Rozet I, Rozet M, Borisovskaya A. Anesthesia for electroconvulsive therapy: an update. Curr Anesthesiol Rep. 2018;8(3):290-297. doi:10.1007/s40140-018-0283-4

11. Jaffe R. The practice of electroconvulsive therapy: recommendations for treatment, training, and privileging: a task force report of the American Psychiatric Association. Am J Psychiatry. 2002;159(2):331-331. doi:10.1176/appi.ajp.159.2.331

12. Molins C, Fortea A, Bioque M, Solé E, Parellada E. Clozapine and electroconvulsive therapy is an effective and safe treatment during pregnancy: a case report. J ECT. 2019;35(3):e30-e32. doi:10.1097/yct.0000000000000617

13. Anderson EL, Reti IM. ECT in pregnancy: a review of the literature from 1941 to 2007. Psychosom Med. 2009;71(2):235-242. doi:10.1097/PSY.0b013e318190d7ca

14. Haghighi M, Sedighinejad A, Naderi Nabi B, et al. The incidence and predictors of headache and myalgia in patients after electroconvulsive therapy (ECT). Anesth Pain Med. 2016;6(3):e33724. doi:10.5812/aapm.33724

15. Serim B, Ulaş H, Ozerdem A, Alkin T. Electroconvulsive therapy in an adolescent pregnant patient. Prog Neuropsychopharmacol Biol Psychiatry. 2010;34(3):546547. doi:10.1016/j.pnpbp.2009.11.014

16. Calaway K, Coshal S, Jones K, Coverdale J, Livingston 
R. A systematic review of the safety of electroconvulsive therapy use during the first trimester of pregnancy. J ECT. 2016;32(4):230-235. doi:10.1097/yct.0000000000000330

17. Balki M, Castro C, Ananthanarayan C. Status epilepticus after electroconvulsive therapy in a pregnant patient. Int J Obstet Anesth. 2006;15(4):325-328. doi:10.1016/j. ijoa.2006.01.005

18. Niu K, Liu H, Chen RW, et al. Use of propofol for prevention of post-delivery nausea during cesarean section: a double-blind, randomized, placebo-controlled trial. J Anesth. 2018;32(5):748-755. doi:10.1007/s00540018-2549-x

19. De Asis SJ, Helgeson L, Ostroff R. The use of propofol to prevent fetal deceleration during electroconvulsive therapy treatment. J ECT. 2013;29(4):e57-58. doi:10.1097/ YCT.0b013e318290f9e7

C 2020 The Author(s); This is an open-access article distributed under the terms of the Creative Commons Attribution License (http:// creativecommons.org/licenses/by/4.0), which permits unrestricted use, distribution, and reproduction in any medium, provided the original work is properly cited. 